Short communication

\title{
Metabolic profiling of the tissue-specific responses in mussel Mytilus galloprovincialis towards Vibrio harveyi challenge
}

\author{
Xiaoli Liu ${ }^{\mathrm{a},{ }^{*}}$, Chenglong Ji ${ }^{\mathrm{b}}$, Jianmin Zhao ${ }^{\mathrm{b}}$, Qing Wang ${ }^{\mathrm{b}}$, Fei Li ${ }^{\mathrm{b}}$, Huifeng Wu ${ }^{\mathrm{b}}$ \\ a School of Life Sciences, Ludong University, Yantai 264025, PR China \\ b Laboratory of Coastal Ecotoxicology, Yantai Institute of Coastal Zone Research, Chinese Academy of Sciences, Yantai 264003, PR China
}

\section{A R T I C L E I N F O}

\section{Article history:}

Received 22 January 2014

Received in revised form

7 April 2014

Accepted 27 May 2014

Available online 6 June 2014

\section{Keywords:}

Metabolomics

Mytilus galloprovincialis

Digestive gland

Gill

Vibrio harveyi

\begin{abstract}
A B S T R A C T
Mussel Mytilus galloprovincialis is a marine aquaculture shellfish distributing widely along the coast in north China. In this work, we studied the differential metabolic responses induced by Vibrio harveyi in digestive gland and gill tissues from M. galloprovincialis using NMR-based metabolomics. The differential metabolic responses in the two tissue types were detected, except the similarly altered taurine and betaine. These metabolic responses suggested that $V$. harveyi mainly induced osmotic disruption and reduced energy demand via the metabolic pathways of glucose synthesis and ATP/AMP conversion in mussel digestive gland. In mussel gill tissues, $V$. harveyi basically caused osmotic stress and possible reduced energy demand as shown by the elevated phosphocholine that is involved in one of the metabolic pathways of ATP synthesis from ADP and phosphocholine. The altered mRNA expression levels of related genes (superoxide dismutase with copper and zinc, heat shock protein 90 , defensin and lysozyme) suggested that $V$. harveyi induced clear oxidative and immune stresses in both digestive gland and gill tissues. However, the mRNA expression levels of both lysozyme and defensin in digestive gland were more significantly up-regulated than those in gill from $V$. harveyi-challenged mussel M. galloprovincialis, meaning that the immune organ, digestive gland, was more sensitive than gill. Overall, our results indicated that $V$. harveyi could induce tissue-specific metabolic responses in mussel M. galloprovincialis.
\end{abstract}

() 2014 Elsevier Ltd. All rights reserved.

\section{Introduction}

Vibrios, such as Vibrio anguillarum, Vibrio alginolyticus, Vibrio splendidus, Vibrio furnissii, Vibrio parahaemolyticus, Vibrio tapetis and Vibrio harveyi, are widely distributed in marine environments and main causative pathogens of vibriosis in both fish and shellfish $[1,2]$. As a type of vibrios, $V$. harveyi is a gram-negative bacterium and its outbreak is a causative agent for the massive mortality of marine aquaculture animals including fish, crustacean and mollusk in warm seas [3,4]. Marine bivalves such as scallop Chlamys farreri, oyster Crassostrea gigas, clam Ruditapes philippinarum and mussel Mytilus galloprovincialis are of substantial economic values and therefore widely cultured in China. The blue mussel M. galloprovincialis has a wide geographic distribution and can be consumed as delicious seafood by the residents along the coast in north China. As a filter-feeder, M. galloprovincialis is not only a good bioindicator in marine ecotoxicology $[5,6]$, but also used in immunity studies of

\footnotetext{
* Corresponding author. Tel.: +86 535 6696155; fax: +86 5356696598 .

E-mail address: zlylxl2009@gmail.com (X. Liu).
}

marine aquaculture animals due to its high accumulation and susceptibility to pathogens [7-9]. For example, Canesi et al. found that infections of vibrios (V. splendidus and V. anguillarum) induced oxidative and immune stresses in $M$. galloprovincialis, indicated by significant up-regulations of gene expression levels of immune stress-responsive molecules, including anti-oxidant enzymes (catalase and glutathione transferase), lysozymes and metallothioneins [8].

In many previous immunity studies, researchers focused on the special immune-related molecules and their functions involved in immune network in M. galloprovincialis [7-10]. With the development of system biology techniques (genomics, transcriptomics, proteomics and metabolomics), researchers have applied these -omic approaches to fish and shellfish immunology, which can provide a comprehensive understanding of immune responses of marine aquaculture animals to pathogen challenges [11,12]. Recently, Ji et al. reported the proteomic responses in M.galloprovincialis challenged by another vibrio, $V$. anguillarum [12]. They found that the infection of $V$. anguillarum caused disruption in energy metabolism and oxidative stress in M. galloprovincialis, indicated by the proteomic biomarkers, including procollagen-proline dioxygenase, protein disulfide 
isomerase, nucleoside diphosphate kinases, electron transfer flavoprotein and glutathione S-transferase [12]. In our previous work, we confirmed that $V$. anguillarum caused more severe oxidative and immune stresses in the digestive gland from clam Ruditapes philippinarum than $V$. splendidus using NMR-based metabolomics [13].

As an immune organ, the digestive gland of marine bivalves is the most frequently used organ in immunity studies due to the filter-feeding habit of $M$. galloprovincialis [8,11,13]. The organ of gill can be directly attacked by marine pathogens and has been also investigated in shellfish immunology $[12,14,15]$. In this work, the metabolic profiles were profiled in both digestive gland and gill tissues from mussel $M$. galloprovincialis challenged by $V$. harveyi. The aim of this work was to illustrate the differential metabolic effects and mechanisms induced by $V$. harveyi in digestive gland and gill tissues of $M$. galloprovincialis using NMR-based metabolomics.

\section{Materials and methods}

\subsection{Animals and experimental conditions}

Adult mussels $M$. galloprovincialis (shell length: $5.0-6.0 \mathrm{~cm}$, $n=45$ ) were collected in July 2013 from an aquaculture farm (Yantai, China). Animals were transported to the laboratory and acclimatized in aerated natural seawater (salinity $31.1 \mathrm{psu}$ ) for $10 \mathrm{~d}$. After acclimatization, the animals were randomly divided into three groups (blank, PBS control and $V$. harveyi challenge) each containing 15 individuals in $30 \mathrm{~L}$ filtered seawater. The culture seawater was renewed daily. During the acclimation period, animals were kept at $25^{\circ} \mathrm{C}$ under a photoperiod of $12 \mathrm{~h}$ light and $12 \mathrm{~h}$ dark, and fed with the Chlorella vulgaris at a ration of $2 \%$ tissue dry weight daily.

\subsection{Challenge experiment}

The bacterium, V. harveyi, was kindly provided by Prof. Baozhong Liu (Institute of Oceanology, CAS). The bacteria were cultured in liquid $2216 \mathrm{E}$ broth (Tryptone $5 \mathrm{~g} \mathrm{~L}^{-1}$, yeast extract $1 \mathrm{~g} \mathrm{~L}^{-1}$, $\mathrm{C}_{6} \mathrm{H}_{5} \mathrm{Fe} \cdot 5 \mathrm{H}_{2} \mathrm{O} 0.1 \mathrm{~g} \mathrm{~L}^{-1}, \mathrm{pH} 7.6$ ) at $29{ }^{\circ} \mathrm{C}$ and centrifuged at $3000 \mathrm{~g}$ for $5 \mathrm{~min}$ to harvest the bacteria. For challenge experiment, live bacteria of $V$. harveyi were re-suspended in PBS. Each challenged mussel was injected with $V$. harveyi $\left(10^{7} \mathrm{CFU} / \mathrm{mL}\right)$ in $50 \mu \mathrm{L}$ of PBS ( $137 \mathrm{mM} \mathrm{NaCl}, 2.7 \mathrm{mM} \mathrm{KCl}, 10 \mathrm{mM} \mathrm{Na}_{2} \mathrm{HPO}_{4}, 2 \mathrm{mM} \mathrm{KH}_{2} \mathrm{PO}_{4}$, pH 7.4) in the adductor muscle. It should be noted that this concentration of bacteria was used to study the metabolic responses of M. galloprovincialis and not environmentally relevant. The environmental concentration of $V$. harveyi is usually smaller than $10^{6} \mathrm{CFU} / \mathrm{mL}$ [16]. The animals in PBS control group were injected with $50 \mu \mathrm{L}$ PBS. The group without any treatment was used as the blank group. For injection, each mussel was poked slightly at the edge of the shells. Then the PBS or bacteria solution was injected carefully into the adductor muscle using a syringe. After injection, all mussels were put back to the tanks and sampled at $24 \mathrm{~h}$ postinjection. The digestive gland and gill tissues of each mussel were dissected quickly and flash-frozen in liquid nitrogen, and then stored at $-80^{\circ} \mathrm{C}$ before RNA and metabolite extraction. For further procedures, a total of 15 individual mussels in each group (blank, PBS control and $V$. harveyi challenge) were used for metabolomics analysis.

\subsection{Metabolite extraction}

Polar metabolites in mussel digestive gland and gill tissues ( $n=15$ for each treatment) were extracted by the modified extraction protocol as described previously [17,18]. Briefly, the tissue $(100 \mathrm{mg}$ ) was homogenized in $400 \mu \mathrm{L}$ of methanol and $85 \mu \mathrm{L}$ of water. Then the mixture was shaken and centrifuged ( $5 \mathrm{~min}$, $3000 \mathrm{~g}$, at $4{ }^{\circ} \mathrm{C}$ ), and the supernatant substance was transferred into a glass vial. A total of $200 \mu \mathrm{L}$ of chloroform and $440 \mu \mathrm{L}$ of water were added to the supernatant, and the mixture was vortexed and centrifuged again $\left(10 \mathrm{~min}, 3000 \mathrm{~g}, 4^{\circ} \mathrm{C}\right)$. The methanol/water layer with polar metabolites was transferred to a glass vial and dried in a centrifugal concentrator. The extracts of tissues were then resuspended in $600 \mu \mathrm{L}$ of phosphate buffer $\left(100 \mathrm{mM} \mathrm{Na}_{2} \mathrm{HPO}_{4}\right.$ and

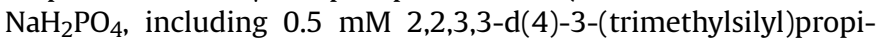
onic acid sodium salt (TSP) as the internal standard, $\mathrm{pH}$ 7.0) in $\mathrm{D}_{2} \mathrm{O}$. The mixture was vortexed and centrifuged at $3000 \mathrm{~g}$ for $5 \mathrm{~min}$ at $4{ }^{\circ} \mathrm{C}$. The supernatant substance $(550 \mu \mathrm{L})$ was then pipetted into a $5 \mathrm{~mm}$ NMR tube prior to NMR analysis.

\subsection{RNA extraction and quantitation of gene expressions}

Total RNA from mussel digestive gland and gill tissues was extracted following the manufacturer's directions (Invitrogen, LifeTechnologies, Carlsbad, CA, USA), and the first-strand cDNA was synthesized according to M-MLV RT Usage information (Promega, Madison, WI, USA). The expressions of the housekeeping genes in M. galloprovincialis were determined by real-time quantitative PCR (qRT-PCR), the data were analyzed with geNorm to calculate the expression stability of the genes ( $M$ values) and the optimal number of reference genes required for accurate normalization ( $V$ values) [19]. GeNorm identified $\beta$-actin as the most stable gene which was then used as the internal control for gene expression normalization (data not shown).

Gene-specific primers (Table 1) for selected genes were used to amplify amplicons specific for $M$. galloprovincialis. The fluorescent qRT-PCR amplifications were carried out in triplicate in a total volume of $20 \mu \mathrm{L}$ containing $10 \mu \mathrm{L}$ of $2 \times$ SYBR Premix Ex Taq ${ }^{\mathrm{TM}}$ (TaKaRa), $0.4 \mu \mathrm{L}$ of $50 \times$ ROX Reference DYE II, $4.8 \mu \mathrm{L}$ DEPC-treated $\mathrm{H}_{2} \mathrm{O}, 0.4 \mu \mathrm{L}$ of each primer, $4.0 \mu \mathrm{L}$ of $1: 20$ diluted cDNA. The qRTPCR program was as following: $50^{\circ} \mathrm{C}$ for $2 \mathrm{~min}$ and $95^{\circ} \mathrm{C}$ for $10 \mathrm{~min}$, followed by 40 cycles of $94^{\circ} \mathrm{C}$ for $15 \mathrm{~s}, 58{ }^{\circ} \mathrm{C}$ for $45 \mathrm{~s}, 72^{\circ} \mathrm{C}$ for $30 \mathrm{~s}$. Dissociation curve analysis of amplification products was performed at the end of each PCR to confirm that only one PCR product was amplified and detected. After the PCR program, data were analyzed with the ABI 7500 SDS software (Applied Biosystems). To maintain consistency, the baseline was set automatically by the software. The comparative CT method ( $2^{-\Delta \Delta C T}$ method) was used to analyze the expression level of the genes [20]. One-way ANOVA combined with Tukey's test was performed on mRNA expression levels between PBS control and $V$. harveyi-challenged groups, respectively. A $P$ value less than 0.05 was considered statistically significant. The Minitab software (Version 15, Minitab Inc., USA) was used for the statistical analysis.

\section{5. ${ }^{1} \mathrm{H}$ NMR spectroscopy}

Metabolite extracts of tissues from mussels were analyzed on a Bruker AV 500 NMR spectrometer performed at $500.18 \mathrm{MHz}$ (at

Table 1

The list of primers used for the quantification of mRNA expressions by qRT-PCR.

\begin{tabular}{llll}
\hline $\begin{array}{l}\text { Gene } \\
\text { name }\end{array}$ & $\begin{array}{l}\text { Accession } \\
\text { no. }\end{array}$ & Forward primer $\left(5^{\prime}-3^{\prime}\right)$ & Reverse primer $\left(5^{\prime}-3^{\prime}\right)$ \\
\hline Cu/Zn-SOD JN863296.1 & GATGAGACGATCAGCCTTC & GTCCAGCATTACCTCCCTTT \\
HSP90 & AJ586906.3 & GATTGATAGCCTGTCTTAG CCACATTTCTGCTGTTTACT \\
Lysozyme & JQ244771.1 & TGACATCAATGCTAACCCAC TTGTATGCAGCTACTCCACC \\
Defensin & JN935272.1 & TCAGATTGAAGTGCAGATG & GAAAACTAAGTGGATGA \\
& & AAGG \\
\hline
\end{tabular}


$25{ }^{\circ} \mathrm{C}$ ) as described previously [21]. All ${ }^{1} \mathrm{H}$ NMR spectra were phased, baseline-corrected, and calibrated (TSP at $0.0 \mathrm{ppm}$ ) manually using TopSpin (version 2.1, Bruker).

\subsection{Spectral pre-processing and multivariate data analysis}

All one dimensional ${ }^{1} \mathrm{H}$ NMR spectra were converted to a data matrix using the custom-written ProMetab software in Matlab version 7.0 (The MathsWorks, Natick, MA, USA) [22]. Each spectrum was segmented into bins with a width of $0.005 \mathrm{ppm}$ between 0.2 and $10.0 \mathrm{ppm}$. The bins of residual water peak between 4.70 and $5.20 \mathrm{ppm}$ were excluded from all the NMR spectra. The total spectral area of the remaining bins was normalized to unity to facilitate the comparison between the spectra. All the NMR spectra were generalized log transformed with a transformation parameter $\lambda=2.0 \times 10^{-9}$ to stabilize the variance across the spectral bins and to increase the weightings of the less intense peaks [22]. Data were mean-centered before multivariate data analysis.

The supervised multivariate data analysis methods, partial least squares discriminant analysis (PLS-DA) and orthogonal projection to latent structure with discriminant analysis (O-PLS-DA), were sequentially carried out to uncover and extract the statistically significant metabolite variations related to $V$. harveyi challenges. The results were visualized in terms of scores plots to show the classifications and corresponding loadings plots to show the NMR spectral variables contributing to the classifications. The model coefficients were calculated from the coefficients incorporating the weight of the variables in order to enhance interpretability of the model and the value of $Q^{2}$ was used to describe the predictive ability of the constructed model and assess the model quality. Then metabolic differences responsible for the classifications between control and $V$. harveyi-challenged groups could be detected in the coefficient-coded loadings plots. The details regarding data analysis were described previously [23]. Metabolites were assigned following the tabulated chemical shifts [24] and the software, Chenomx (Evaluation Version, Chenomx Inc., Edmonton, Alberta, Canada).

\section{Results and discussion}

3.1. Metabolic differences in digestive gland and gill tissues from M. galloprovincialis from PBS control group

As a system biology technique, metabolomics has been widely employed in multiple areas, including toxicology and immunology [11,13,25-30]. Fig. 1 indicates the ${ }^{1} \mathrm{H}$ NMR spectra of tissue extracts of digestive gland (Fig. 1A) and gill (Fig. 1B) samples from PBS control mussel group. The identified metabolites, including amino acids (branched chain amino acids: valine, leucine and isoleucine, aspartate, glutamate, glutamine, tyrosine phenylalanine, etc.), energy storage compounds (ATP, glucose and glycogen), an intermediate in Krebs cycle (succinate), osmolytes (betaine, taurine, glycine and homarine) and a phosphagen (phosphocholine), were labeled in Fig. 1. As shown in Fig. 1, both ${ }^{1} \mathrm{H}$ NMR spectra are dominated by the organic osmolyte, betaine (3.27 and $3.90 \mathrm{ppm}$ ), which is synthesized from a two-step reaction of choline: choline $\rightarrow$ betaine aldehyde $+\mathrm{NAD}^{+} \rightarrow$ betaine $+\mathrm{NADH}$ [31]. Since digestive gland is the main organ of glucose and protein metabolisms, the ${ }^{1} \mathrm{H}$ NMR spectrum from the digestive gland sample should present the higher levels of most amino acids and glucose than those in the ${ }^{1} \mathrm{H}$ NMR spectrum from the gill sample. Initially, O-PLS-DA was performed on the NMR spectral datasets from the blank and PBS control groups. However, no significant separation between blank and PBS groups was found (data not shown), which suggested that the single injection of PBS in the adductor muscle did not obviously

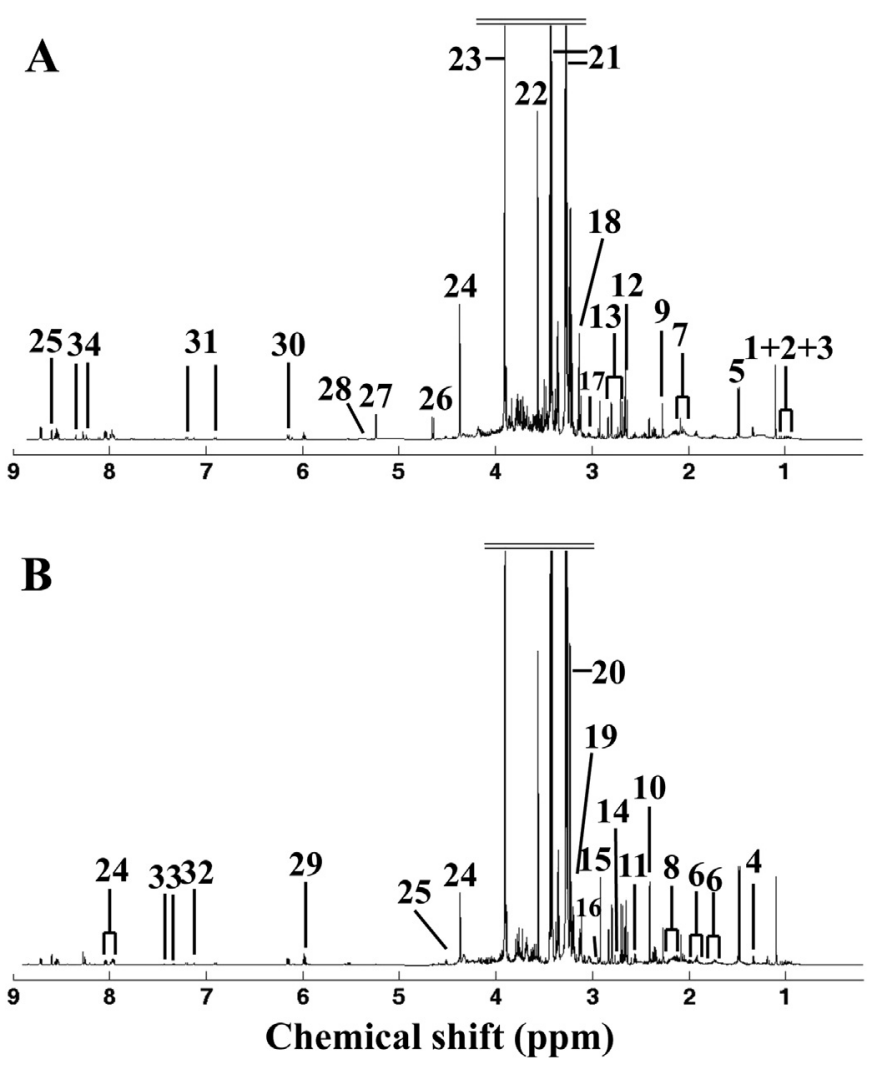

Fig. 1. Typical 1-dimensional $500 \mathrm{MHz}{ }^{1} \mathrm{H}$ NMR spectra of tissue extracts of digestive gland (A) and gill (B) from one PBS control mussel M. galloprovincialis. Keys: (1) leucine, (2) isoleucine, (3) valine, (4) threonine, (5) alanine, (6) arginine, (7) glutamate, (8) glutamine, (9) acetoacetate, (10) succinate, (11) $\beta$-alanine, (12) hypotaurine, (13) aspartate, (14) dimethylamine, (15) dimethylglycine, (16) asparagine, (17) lysine, (18) malonate, (19) choline, (20) phosphocholine, (21) taurine, (22) glycine, (23) betaine, (24) homarine, (25) AMP, (26) $\beta$-glucose, (27) $\alpha$-glucose, (28) glycogen, (29) unknown 1 (5.95 ppm), (30) ATP, (31) tyrosine, (32) histidine, (33) phenylalanine and (34) adenosine.

affect the metabolic profiles in both digestive gland and gill tissues. Therefore, only PBS control was used in further data analysis. OPLS-DA analysis resulted in a clear separation between the digestive gland and gill samples from PBS control group (Fig. 2A), with a $Q^{2}$ value greater than 0.9 showing the robustness of the constructed model, which confirmed the significant metabolic differences between these two tissues. Clearly, the corresponding loading plot (Fig. 2B) displayed the higher levels of most amino acids (threonine, alanine, glutamate, glutamine, etc.) and glucose, as well as the higher levels of taurine and homarine and lower levels of aspartate and betaine in digestive glands than those in gills. These biological (metabolic) differences between digestive gland and gill tissues suggested the potential differential metabolic responses in these two organs towards $V$. harveyi challenge.

\subsection{Differential metabolic responses in digestive gland and gill tissues from $M$. galloprovincialis challenged by $V$. harveyi}

O-PLS-DA was conducted on the ${ }^{1} \mathrm{H}$ NMR spectral data to characterize the metabolic responses in digestive gland and gill tissue samples from PBS control group, respectively (Fig. 3). Fig. 3A and C indicated that O-PLS-DA resulted in clear classifications between PBS control and $V$. harveyi-challenged mussel groups, respectively, with reliable $Q^{2}$ values (>0.7). From the loading plot (Fig. 3B), the concentrations of adenosine and AMP were significantly $(P<0.05)$ increased in $V$. harveyi-challenged mussel digestive gland. The 


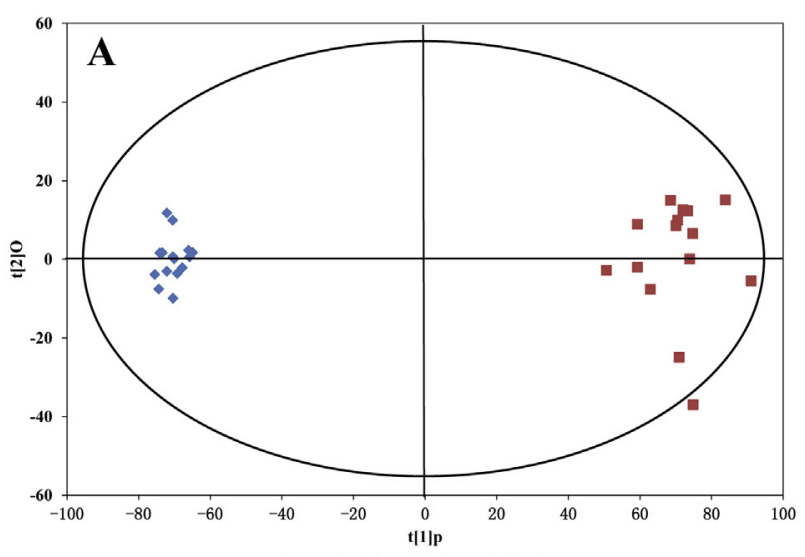

$R^{2} X=80.4 \%, Q^{2}=0.98$

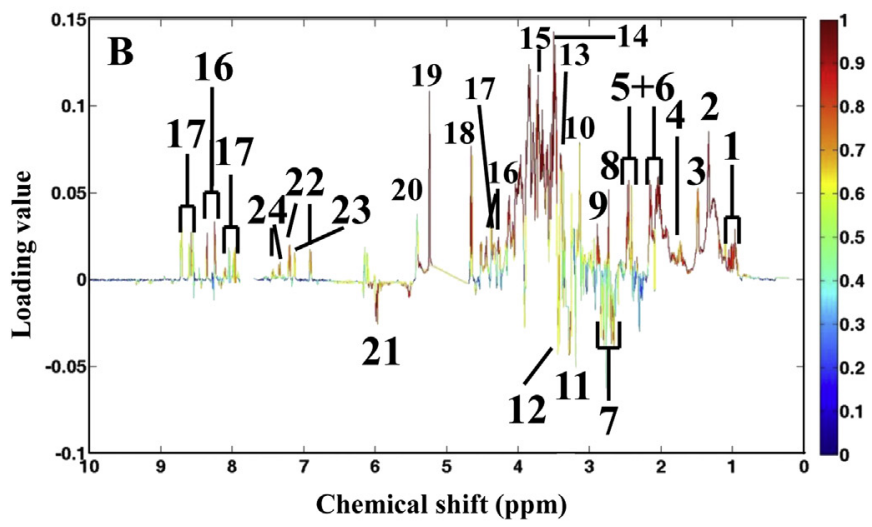

Fig. 2. O-PLS-DA scores plot (A) derived from ${ }^{1} \mathrm{H}$ NMR spectra of tissue extracts of digestive gland $(\square)$ and gill ( $\downarrow$ ) from PBS control group and corresponding coefficient plot (B). The color map shows the significance of metabolite variations between the two classes (digestive gland and gill). Peaks in the positive direction indicate metabolites that are more abundant in digestive gland tissues. Consequently, metabolites that are more abundant in gill tissues are presented as peaks in the negative direction. Keys: (1) branched chain amino acids: leucine, isoleucine and valine, (2) threonine, (3) alanine, (4) arginine, (5) glutamate, (6) glutamine, (7) aspartate, (8) dimethylamine, (9) dimethylglycine, (10) lysine, (11) malonate, (12) choline, (13) phosphocholine, (14) taurine, (15) glycine, (16) adenosine, (17) homarine, (18) $\beta$-glucose, (19) $\alpha$-glucose, (20) glycogen, (21) unknown 1 (5.95 ppm), (22) tyrosine, (23) histidine and (25) phenylalanine.

levels of aspartate, dimethylglycine, glucose, ATP, taurine and betaine were significantly $(P<0.05)$ decreased. Adenosine plays important roles in both energy transfer as adenosine triphosphate (ATP) and adenosine diphosphate (ADP), as well as in signal transduction as cyclic adenosine monophosphate [32]. In the digestive gland from $V$. harveyi-challenged mussels, the depleted ATP meant a reduced energy demand resulting in the elevated adenosine and depleted glucose. Apparently, the increased AMP was resulted in the enhanced hydrolysis of ATP, which was also observed in V. splendidus-challenged sea cucumber Apostichopus japonicas [33]. Dimethylglycine, taurine and betaine are osmolytes in animals. The decreased dimethylglycine, taurine and betaine probably indicated the osmotic stress induced by $V$. harveyi challenge in mussel digestive gland. The level of aspartate was significantly decreased in $V$. harveyi-challenged mussel digestive gland tissues. In marine mollusks, amino acids can be used to regulate animals' intracellular osmolarity with the environment and also can be oxidized to generate energy [34]. In the energy metabolic pathway, there is substantial conversion of aspartate to succinate with no detectable enrichment of other metabolites under anoxic conditions in mollusk [35]. However, both ATP and glucose were decreased and succinate was not altered in $V$. harveyi-challenged mussel digestive gland. Therefore, the decrease of aspartate could be related to the disruption in osmotic regulation but in energy metabolisms in $V$. harveyi-challenged mussel digestive gland. In a previous study on the effects of $V$. harveyi challenge towards clam Ruditapes philippinarum, the elevation of branched chain amino acids (BCAA) was observed in digestive gland of $R$. philippinarum [36]. In the metabolic pathways of innate immunity, BCAAs have an availability on the immune system to function by incorporating BCAA into proteins, resulting in a remarkable increase in demand for BCAA for substrates by the immune system [37]. In this work, however, BCAAs were not altered in $V$. harveyi-challenged mussel digestive gland, which suggested the differential responsive mechanisms between mussel $M$. galloprovincialis and $R$. philippinarum to $V$. harveyi challenge.

The metabolic responses in the gill tissues of $V$. harveyi-challenged mussels were different from those in the digestive gland samples. As shown in the corresponding loading plot of O-PLS-DA (Fig. 3D), phosphocholine, hypotaurine, glycine, homarine and an unknown metabolite $(1.10 \mathrm{ppm})$ were increased in $V$. harveyichallenged mussel gill samples (Fig. 3D). Two organic osmolytes, taurine and betaine were significantly $(P<0.05)$ decreased, which was similar to their alteration in digestive gland of $V$. harveyichallenged mussels (Fig. 3B). The elevated organic osmolytes, including hypotaurine, homarine and glycine, clearly indicated the osmotic stress caused by $V$. harveyi challenge in the gill of mussel $M$. galloprovincialis. However, other two osmolytes, taurine and betaine were depleted. Since taurine can synthesized into hypotaurine, the decrease of taurine meant the enhanced synthesis of hypotaurine from taurine, resulting in an increase of hypotaurine in $V$. harveyi-challenged mussel gill samples. Similarly, betaine is synthesized from glycine. Therefore, the depletion of betaine combined with the elevation of glycine indicated the reduced synthesis of betaine $V$. harveyi-challenged mussel gill samples. Ji et al. reported the similar metabolic responses including depleted taurine and betaine and elevated homarine in gill tissues from mussel $M$. galloprovincialis challenged by $V$. anguillarum, which implied the similar disturbance in osmotic regulation induced by both $V$. anguillarum and $V$. harveyi in mussel gills [12]. As a phosphagen, phosphocholine is involved in the conversion of ATP and choline into phosphocholine and ADP, which is catalyzed by choline kinase. Therefore, the elevated phosphocholine probably meant a reduced energy demand in $V$. harveyi-challenged mussel gills via a different metabolic pathway compared with that in digestive gland, as mentioned above.

\subsection{Stress-responsive gene expressions in digestive gland and gills from M. galloprovincialis challenged by $V$. harveyi}

In this study, four stress-responsive genes including heat shock protein 90 (HSP90), superoxide dismutase with copper and zinc ( $\mathrm{Cu} / \mathrm{Zn}-\mathrm{SOD})$, defensin and goose-type lysozyme related to diverse functions were used for the quantification of mRNA expressions. HSP90, $\mathrm{Cu} / \mathrm{Zn}-\mathrm{SOD}$, defensin and lysozyme play important roles in anti-oxidative stress and immune defense. After $V$. harveyi injection, the mRNA expression levels of HSP90, Cu/Zn-SOD, defensin and lysozyme mRNA in both digestive gland and gill tissues were quantified using qRT-PCR technique with $\beta$-actin as internal control (Fig. 4). All the mRNA expression levels of these four selected stressresponsive genes were significantly $(P<0.05)$ up-regulated in $V$. harveyi-challenged mussel digestive gland and gill tissues (Fig. 4). $\mathrm{Cu} / \mathrm{Zn}$-SOD is a known anti-oxidant enzyme involved in scavenging reactive oxygen species and HSP90 belongs to a large family of molecular chaperones playing vital roles in preventing irreversible protein denaturation, aggregation and misfolding $[38,39]$. They can be induced by oxidative stress and pathogen infection [38]. Both 

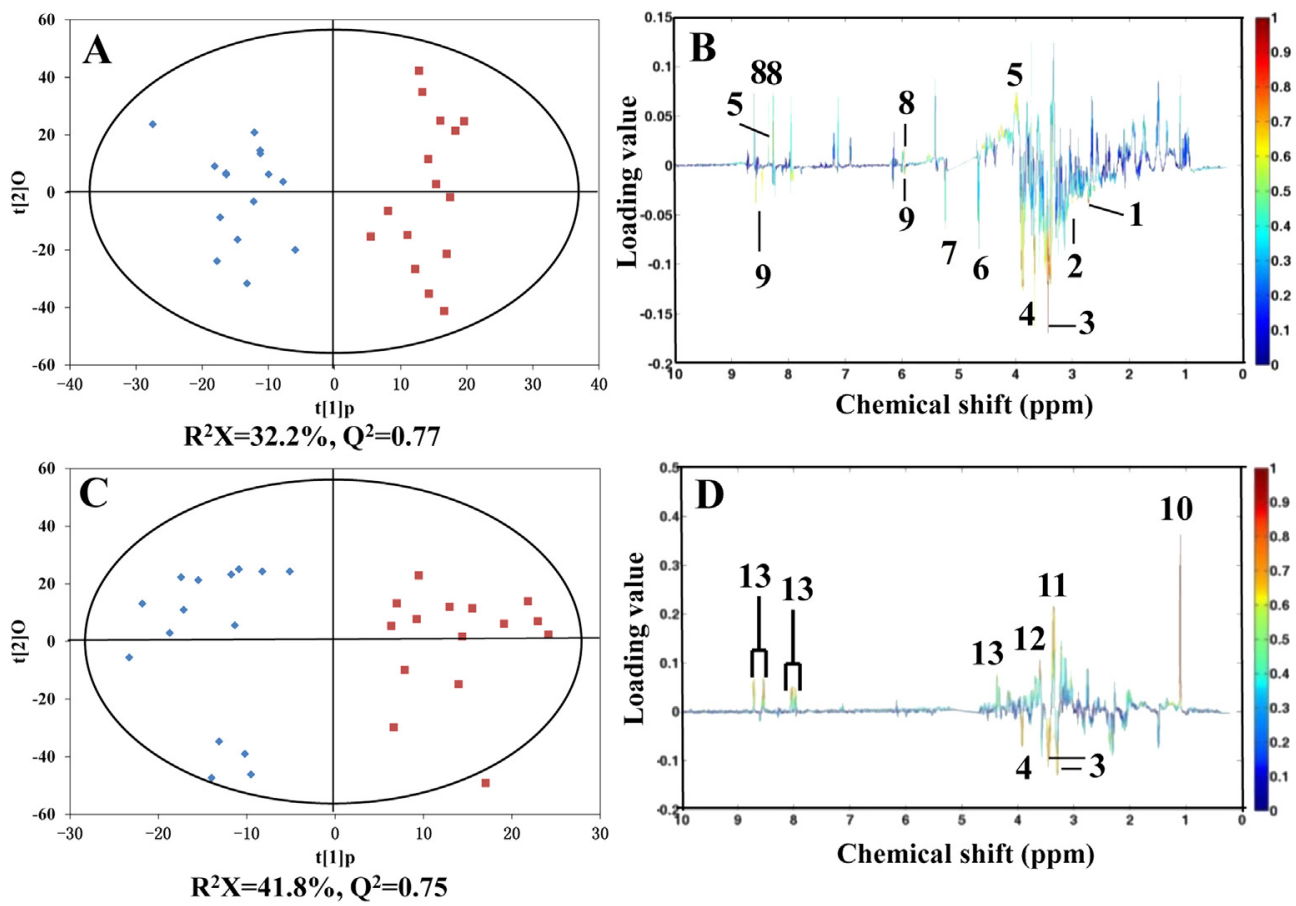

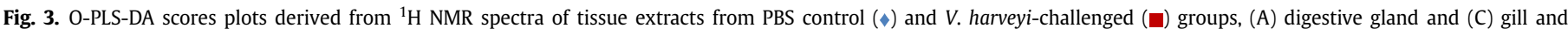

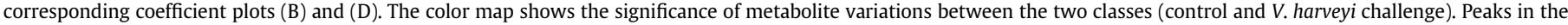

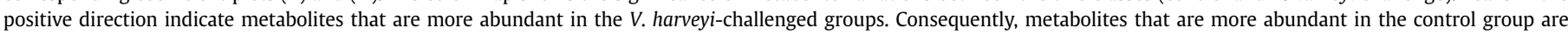

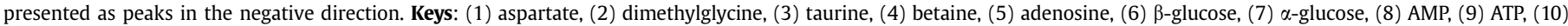
unknown 1 (1.10 ppm), (11) phosphocholine, (12) hypotaurine, (13) glycine and (14) homarine.

defensin and lysozyme are antibacterial components that have been characterized in marine mollusks $[40,41]$. In this work, the significant up-regulation of mRNA expression levels of $\mathrm{Cu} / \mathrm{Zn}-\mathrm{SOD}$, HSP90, defensin and lysozyme indicated the oxidative and immune stresses induced by $V$. harveyi in both digestive gland and gill samples from $V$. harveyi-challenged mussels. In addition, the mRNA expression levels of defensin and lysozyme were more significantly
$(P<0.05)$ up-regulated in digestive gland than those in gill tissue samples. This might imply that the immune system in digestive glands was more sensitive than that in gills from $V$. harveyi-challenged mussels, since the digestive gland is the main immune organ in mussel M. galloprovincialis.

In summary, the differential metabolic responses induced by $V$. harveyi in digestive gland and gill tissues from $M$. galloprovincialis
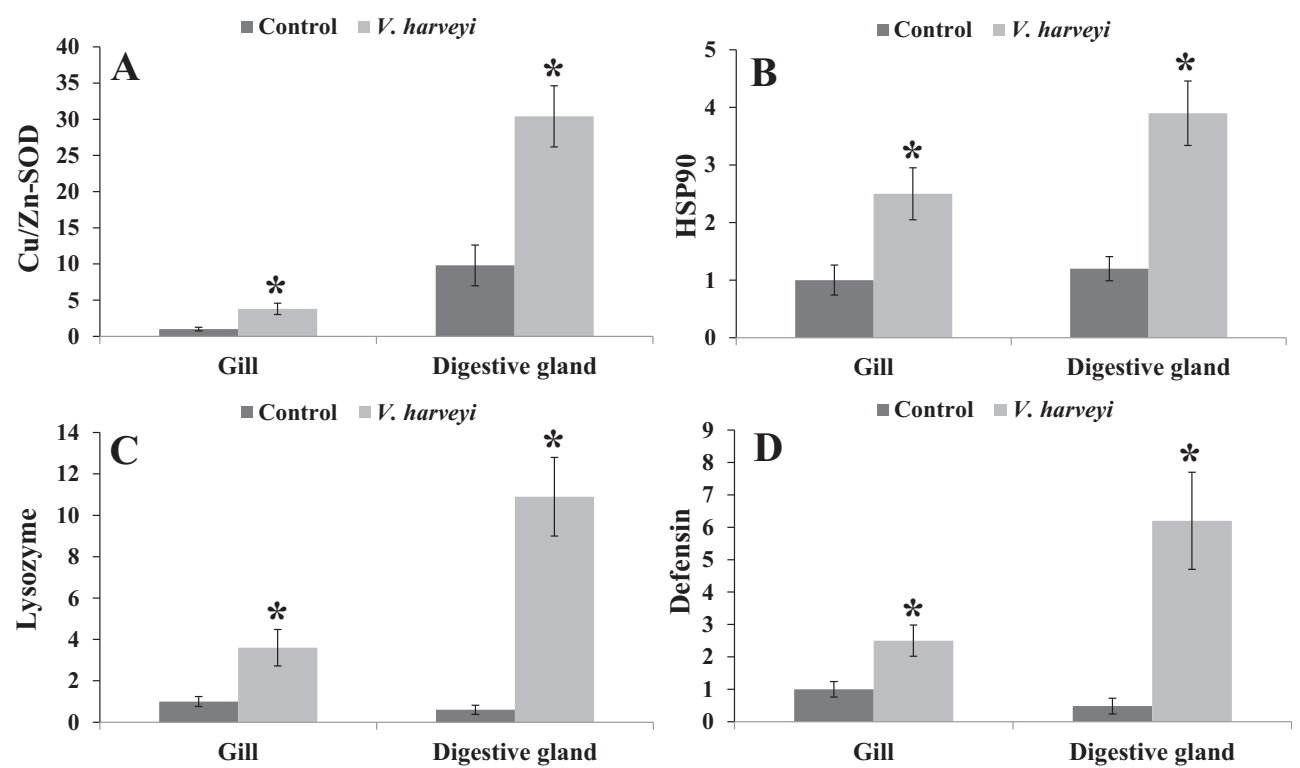

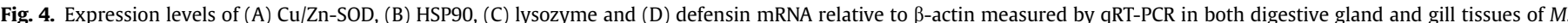

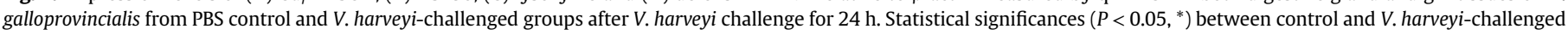
M. galloprovincialis samples were determined by one-way ANOVA. Abbreviations: Cu/Zn-SOD, superoxide dismutase with copper and zinc and HSP90, heat shock protein 90 . 
were investigated using NMR-based metabolomics. Overall, $V$. harveyi induced osmotic disruption and reduced energy demand via the metabolic pathways of glucose synthesis and ATP/AMP conversion in mussel digestive gland. In mussel gill tissues, $V$. harveyi basically caused osmotic stress and possible reduced energy demand through the metabolic pathway of conversion of phosphocholine and ADP to choline and ATP. The altered mRNA expression levels of related genes $(\mathrm{Cu} / \mathrm{Zn}-\mathrm{SOD}$, HSP90, defensin and lysozyme) suggested that $V$. harveyi induced clear oxidative and immune stresses in both digestive gland and gill tissues. However, the mRNA expression levels of both lysozyme and defensin in digestive gland were more significantly up-regulated than those in gill from $V$. harveyi-challenged mussel $M$. galloprovincialis. This work demonstrated that $V$. harveyi could induce tissue-specific metabolic responses in the digestive gland and gill tissues from mussel M. galloprovincialis using NMR-based metabolomics.

\section{Acknowledgment}

This work was supported by A Project of Shandong Province Higher Educational Science and Technology Program (No. J14LE08). The authors thank Prof. Mark Viant for the use of ProMetab software.

\section{References}

[1] Yang S, Wang Y, Dong S. Progress of research on vibriosis in marine cultured fish (In Chinese). Mar Fish Res 2005;26:75-83.

[2] Wu X, Pan J, Jian J. Advances in studies on shellfish diseases: on microbial diseases of shellfish. Mar Sci Bull 1995;14:82-91.

[3] Austin B, Zhang XH. Vibrio harveyi: a significant pathogen of marine vertebrates and invertebrates. Lett Appl Microbiol 2006;43(2):119-24.

[4] Travers M-A, Le Goic N, Huchette S, Koken M, Paillard C. Summer immune depression associated with increased susceptibility of the European abalone, Haliotis tuberculata to Vibrio harveyi infection. Fish Shellfish Immunol 2012;32:170-7.

[5] Ciacci C, Barmo C, Gallo G, Maisano M, Cappello T, Agata A, et al. Effects of sublethal, environmentally relevant concentrations of hexavalent chromium in the gills of Mytilus galloprovincialis. Aquat Toxicol 2012;120-121:109-18.

[6] Li A, Ma J, Cao J, McCarron P. Toxins in mussels (Mytilus galloprovincialis) associated with diarrhetic shellfish poisoning episodes in China. Toxicon 2012;60:420-5.

[7] Costa MM, Prado-Alvarez M, Gestal C, Li H, Roch P, Novoa B, et al. Functional and molecular immune response of Mediterranean mussel (Mytilus galloprovincialis) haemocytes against pathogen-associated molecular patterns and bacteria. Fish Shellfish Immunol 2009;26:515-23.

[8] Canesi L, Barmo C, Fabbri R, Ciacci C, Vergani L, Roch P, et al. Effects of vibrio challenge on digestive gland biomarkers and antioxidant gene expression in Mytilus galloprovincialis. Comp Biochem Physiol Part C 2010;152:399-406.

[9] Cellura C, Toubiana M, Parrinello N, Roch P. HSP70 gene expression in Mytilus galloprovincialis hemocytes is triggered by moderate heat shock and Vibrio anguillarum, but not by V. splendidus or Micrococcus lysodeikticus. Dev Comp Immunol 2006;30:984-97.

[10] Li H, Parisi MG, Toubiana M, Cammarata M, Roch P. Lysozyme gene expression and hemocyte behaviour in the Mediterranean mussel, Mytilus galloprovincialis, after injection of various bacteria or temperature stresses. Fish Shellfish Immunol 2008;25:143-52.

[11] Wu H, Ji C, Wei L, Zhao J, Lu H. Proteomic and metabolomic responses in hepatopancreas of Mytilus galloprovincialis challenged by Micrococcus luteus and Vibrio anguillarum. J Proteomics 2013;94:54-67.

[12] Ji C, Wu H, Wei L, Zhao J, Wang Q, Lu H. Responses of Mytilus galloprovincialis to bacterial challenges by metabolomics and proteomics. Fish Shellfish Immunol 2013;35:489-98.

[13] Liu X, Ji C, Zhao J, Wu H. Differential metabolic responses of clam Ruditapes philippinarum to Vibrio anguillarum and Vibrio splendidus challenges. Fish Shellfish Immunol 2013;35:2001-7.

[14] Matozzo V, Giacomazzo M, Finos L, Marin MG, Bargelloni L, Milan M. Can ecological history influence immunomarker responses and antioxidant enzyme activities in bivalves that have been experimentally exposed to contaminants? A new subject for discussion in "eco-immunology" studies Fish Shellfish Immunol 2013;35:126-35.

[15] Luna-Acosta A, Saulnier D, Pommier M, Haffner P, De Decker S, Renault T, et al. First evidence of a potential antibacterial activity involving a laccase-type enzyme of the phenoloxidase system in Pacific oyster Crassostrea gigas haemocytes. Fish Shellfish Immunol 2011;31:795-800.

[16] Zhang X, Robertson P, Feng J, Ji W, Austin B. Detection of Vibrio harveyi in larval rearing water of Penaeus chinensis (In Chinese). J Ocean Univ Qingdao $1998 ; 28: 70-4$

[17] Wu H, Southam AD, Hines A, Viant MR. High throughput tissue extraction protocol for NMR and mass spectrometry based metabolomics. Anal Biochem 2008;372:204-12.

[18] Lin CY, Wu H, Tjeerdema RS, Viant MR. Evaluation of metabolite extraction strategies from tissue samples using NMR metabolomics. Metabolomics 2007:3:55-67.

[19] Dang W, Sun L. Determination of internal controls for quantitative real time RT-PCR analysis of the effect of Edwardsiella tarda infection on gene expression in turbot (Scophthalmus maximus). Fish Shellfish Immunol 2011;30: $720-8$.

[20] Livak KJ, Schmittgen TD. Analysis of relative gene expression data using realtime quantitative PCR and the 2(-Delta Delta C(T)) method. Methods 2001;25: 402-8.

[21] Liu X, Zhang L, You L, Yu J, Zhao J, Li L, et al. Differential toxicological effects induced by mercury in gills from three pedigrees of Manila clam Ruditapes philippinarum by NMR-based metabolomics. Ecotoxicology 2011;20:177-86.

[22] Parsons HM, Ludwig C, Gunther UL, Viant M. Improved classification accuracy in 1- and 2-dimensional NMR metabolomics data using the variance stabilising generalised logarithm transformation. BMC Bioinformatics 2007;8:234.

[23] Feng J, Li J, Wu H, Chen Z. Metabolic responses of HeLa cells to silica nanoparticles by NMR-based metabolomic analyses. Metabolomics 2013;9: 874-86.

[24] Fan WMT. Metabolite profiling by one- and two-dimensional NMR analysis of complex mixtures. Prog Nucl Magn Reson 1996;28:161-219.

[25] Liu X, Zhang L, You L, Cong M, Zhao J, Wu H, et al. Toxicological responses to acute mercury exposure for three species of Manila clam Ruditapes philippinarum by NMR-based metabolomics. Environ Toxicol Pharmacol 2011;31: 323-32.

[26] Li Z, Wu H, Zhang X, Li X, Liao P, Li W, et al. Investigation on the acute biochemical effects of light rare earths (lanthanum and cerium) by NMRbased metabonomic approaches. Chem J Chin Univ 2006;27:438.

[27] Williams TD, Turan N, Diab AM, Wu H, Mackenzie C, Bartie KL, et al. Towards a system level understanding of non-model organisms sampled from the environment: a network biology approach. PLoS Comput Biol 2011;7: e1002126.

[28] Wu H, Zhang X, Li X, Wu Y, Pei F. Acute biochemical effects of $\mathrm{La}\left(\mathrm{NO}_{3}\right)_{3}$ on liver and kidney tissues by magic-angle spinning ${ }^{1} \mathrm{H}$ nuclear magnetic resonance spectroscopy and pattern recognition. Anal Biochem 2005;339:242-8.

[29] Wu H, Zhang X, Wu Y, Pei F. Studies on the acute biochemical effects of $\mathrm{La}\left(\mathrm{NO}_{3}\right)_{3}$ using ${ }^{1} \mathrm{H}$ NMR spectroscopy of urine combined with pattern recognition. J Inorg Biochem 2005;99:644-51.

[30] Wu H, Wang W-X. NMR-based metabolomic studies on the toxicological effects of cadmium and copper on green mussels Perna viridis. Aquat Toxicol 2010;100:339-45.

[31] Ferrino LA, Pierce SK. Betaine aldehyde dehydrogenase kinetics partially account for oyster population differences in glycine betaine synthesis. J Exp Zool 2000;286:238-49.

[32] Jackson TC, Mi Z, Jackson EK. Modulation of cyclic AMP production by signal transduction pathways in preglomerular microvessels and microvascular smooth muscle cells. J Pharmacol Exp Ther 2004;310:349-58.

[33] Shao Y, Li C, Ou C, Zhang P, Lu Y, Su X, et al. Divergent metabolic responses of Apostichopus japonicus suffered from skin ulceration syndrome and pathogen challenge. J Agric Food Chem 2013 Nov 13;61(45):10766-71.

[34] Viant MR, Rosenblum ES, Tjeerdema RS. NMR-based metabolomics: a powerful approach for characterizing the effects of environmental stressors on organism health. Environ Sci Technol 2003;37:4982-9.

[35] Graham RA, Ellington WR. Anaerobic aspartate metabolism and the formation of alanine in molluscan cardiac muscle: a ${ }^{13}$ C NMR study. J Exp Zool 1985;236: 365-70.

[36] Liu X, Zhao J, Wu H, Wang Q. Metabolomic analysis revealed the differential responses in two pedigrees of clam Ruditapes philippinarum towards Vibrio harveyi challenge. Fish Shellfish Immunol 2013;35:1969-75.

[37] Calder PC. Branched chain amino acids and immunity. J Nutr 2006;136: 288S-93S.

[38] Wang X, Wang L, Yao C, Qiu L, Zhang H, Zhi Z, et al. Alternation of immune parameters and cellular energy allocation of Chlamys farreri under ammoniaN exposure and Vibrio anguillarum challenge. Fish Shellfish Immunol 2012;32: $741-9$.

[39] Hartl FU, Hayer-Hartl M. Molecular chaperones in the cytosol: from nascent chain to folded protein. Science 2002;295:1852-8.

[40] Zhao J, Li C, Chen A, Li L, Su X, Li T. Molecular characterization of a novel big defensin from clam Venerupis philippinarum. PLoS One 2010;5:e13480.

[41] Zhao J, Qiu L, Ning X, Chen A, Wu H, Li C. Cloning and characterization of an invertebrate type lysozyme from Venerupis philippinarum. Comp Biochem Physiol B Biochem Mol Biol 2010;156:56-60. 